NOTA CIENTÍFICA

\title{
Viscachataenia quadrata Denegri, Dopchiz, Elissondo \& Beveridge, 2003 (Cestoda: Anoplocephalidae) en el Perú
}

\section{Viscachataenia quadrata Denegri, Dopchiz, Elissondo \& Beveridge, 2003 (Cestoda: Anoplocephalidae) in Peru}

1 Laboratorio de Parasitología. Facultad de Medicina Veterinaria y Zootecnia. Universidad Peruana Cayetano Heredia. Lima, Perú Email Manuel Tantaleán: mtantaleánv@hotmail.com

2 Departamento de Protozoología, Helmintología e Invertebrados Afines. Museo de Historia Universidad Nacional Mayor de San Marcos. Lima, Perú. Apartado 14-0434, Lima-14, Perú. Email Lidia Sánchez: lidiarosa06@yahoo.es

Presentado: 07/05/2009 Aceptado: 10/06/2009 Publicado online: $28 / 08 / 2009$
Manuel Tantaleán ${ }^{1}$, Lidia Sánchez ${ }^{2}$ y Patricia Salízar ${ }^{2}$

\section{Resumen}

Se identifica a Viscachataenia quadrata Denegri, Dopchiz, Elissondo \& Beveridge, 2003 obtenidos del intestino delgado de Lagidium peruanum Meyen, capturados en los departamentos de Lima, Puno y Ayacucho.

Palabras claves: Parásito de vida silvestre, Cestoda, Anoplocephalidae, Lagidium peruanum, fauna andina.

\section{Abstract}

We are identified to Viscachataenia quadrata Denegri, Dopchiz, Elissondo \& Beveridge, 2003 of the small intestine of Lagidium peruanum Meyen, from Lima, Puno and Ayacucho.

Keywords: Parasite wildlife, Cestoda, Anoplocephalidae, Lagidium peruanum, Andean wildlife.
La fauna helmíntica de los mamíferos silvestres, en especial de los pequeños, está insuficientemente conocida en el Perú a pesar de su enorme importancia como parte del ecosistema y de ser considerados como indicadores biológicos de las perturbaciones del medio ambiente por su alta sensibilidad. Existen alrededor de 500 especies de mamíferos, de las cuales 161 corresponden al Orden Rodentia, dentro de éstos, la especie endémica Lagidium peruanum Meyen (Chinchillidae) “viscacha” vive en lugares pedregosos o rocosos, aproximadamente desde cerca del nivel del mar hasta los 4,500 metros de altura y es objeto de caza para la alimentación, por su piel y como trofeo de caza o para elaborar productos de artesanía.

En el Perú, desde el año 1958, se han colectado varios helmintos de Lagidium peruanum procedente de varias localidades; algunos de ellos se identificaron taxonómicamente y han sido motivo de publicaciones (Parra 1953, Bain \& Hocquet 1968, Buckley 1973), pero otros no lo fueron permaneciendo depositados en la Colección Helmintológica del Museo de Historia Natural de la Universidad de San Marcos o en colecciones particulares. El material archivado está compuesto por nemátodes y céstodes Anoplocephalidae.

Del material de Anoplocephalidae, examinamos los especimenes grandes, colectados del intestino. La identificación corresponde a Viscachataenia quadrata Denegri, Dopchiz, Elissondo \& Beveridge, 2003. El material identificado se encuentra depositado en la Colección Helmintológica del Museo de Historia Natural con los siguientes números de ingresos:

MUSM No 132. Procedencia: Hacienda Checayani, Provincia de Azángaro, Dpto. de Puno, 3850 msnm

MUSM No 963. Procedencia: Carampoma, Provincia de Huarochirí, Departamento de Lima, 3408 msnm

MUSM No 2727. Procedencia: Distrito de Tambo, provincia La Mar, departamento de Ayacucho, sobre los $3000 \mathrm{msnm}$.

Los especímenes fueron coloreados con carmín de Semichón y, antes de montarlos en bálsamo de Canadá, se les retiró el tegumento y parte de la capa muscular de ambas caras del proglótido, para facilitar la visualización de los órganos internos. También se hicieron cortes sagitales y transversales, a mano, utilizando una navaja de afeitar.
Las características de nuestros especímenes coinciden plenamente con aquellas de Viscachataenia quadrata señaladas por Denegri et al. (2003) obtenidos de Lagidium viscachiae procedentes de Argentina.

El género Viscachataenia se caracteriza por tener doble juego de aparato reproductor en cada proglótido, vagina anterior al saco del cirro, útero grávido reticulado y huevos generalmente con 4 lóbulos; estos huevos, cuando se encuentran en las materias fecales, tienen color pardo oscuro y una oncósfera pequeña rodeada de un embrióforo con aparato piriforme, pero carecen de las minúsculas proyecciones en la superficie. La presencia de vagina anterior al saco del cirro, difícil de observar por ser muy delgada en la porción distal, es única entre los anoplocefálidos con doble juego de aparato reproductor, por lo que Denegri et al. (2003) lo relacionan con el género Monoecocestus que tiene un solo juego de aparato reproductor y que presenta la vagina en igual posición. Posiblemente, por la dificultad de establecer la posición de la vagina y por el mal estado del material, esta especie ha sido identificada previamente como Cittotaenia quadrata Linstow 1904, colocada después como especie inquirendae (Beveridge 1978), Pseudocittotaenia (Tenora 1976) o Mosgovoyia viscaciae (Spassky 1951, Beveridge 1978).

En el Perú, la familia Anoplocephalidae Kolodkowsky, 1902 se encuentra representada por 3 subfamilias: Anoplocephalinae Blanchard, 1891 (con 6 géneros), Thysanosomatinae Skrjabin, 1933 (con 2 géneros) y Linstowiinae Führmann, 1907 (con 3 géneros) totalizando 18 especies parásitas de animales domésticos y de vida silvestre; las especies reconocidas en mamíferos domésticos son: Thysanosoma actinioides Diesing, 1835 en Bos taurus y Ovis aries; Thysaniezia giardi (Moniez, 1829) Skrjabin, 1926 en B. taurus, O. aries y Lama pacos; Anoplocephala magna (Abildgaard, 1789) Sprengel, 1905 en Equus caballus y E. asinus; A. perfoliata (Goeze, 1782) Blanchard, 1848 en $E$. caballus; Anoplocephaloides mamillana (Mehlis, 1831) Rausch, 1976 en E. caballus y E. asinus; Moniezia expansa (Rudolphi, 1805) Blanchard, 1891 en B. taurus, Capra hircus, Sus scrofa domestica, Equus caballus, Lama glama, L. pacus y Vicugna vicugna; M. benedeni (Moniez, 1879) Blanchard, 1891 en B.taurus, $O$. aries, L. pacos y V. vicugna (Chávez \& Zaldívar, 1967; Gómez 
et al. 2008, Zaldívar 1991). En mamíferos silvestres se conocen a Monoecocestus parcitesticulatus Rego, 1960 en Cavia aperea; M. thrtelkeldi (Parra, 1953) Beveridge, 1994 y Monoecocestus sp. en Lagidium peruanum; Mathevotaenia megastoma (Diesing, 1850) Spasskii, 1950 en Saimiri boliviensis, S. sciurea y Saguinus nigricollis. Esta es la primera vez que se identifica plenamente a Viscachataenia quadrata de Lagidium peruanum y se menciona su distribución en el Perú.

\section{Literatura citada}

Bain O. \& P. Hocquet. 1968. Ackertia dorsti n. sp., parasite de la viscache Lagidium peruanum. Bulletin du Muséum nacional d'histoire naturelle, ser. 2. 40: 399-402.

Beveridge I. 1978. A taxonomic revision of the genera Cittotaenia Riehm, 1881, Ctenotaenia Railliet, 1893, Mosgovoyia Spasskii, 1951 and Pseudocittotaenia Tenora, 1976 (Cestoda: Anoplocephalidae). Mémoires du Muséum National d'Histoire Naturelle, Paris. Série A, Zoologie 107: 1-64.

Buckley J.C. 1973. A redescription of Dipetalonema finlayi (Mazza and Tiora, 1932) from a viscacha, Lagidium peruanum of Peru. Ann. Parasit. Hum. Comp. 48: 335-341.

Chavez C. \& Zaldívar, R. 1967. Zooparasites of livestock in Peru. U. S. Dept. Agric. Project PG-PE-102. Univ. San Marcos, School of Vet. Med. Lima, Peru.
Denegri G., M. C. Dopchiz, M. C. Elissondo \& I. Beveridge. 2003. Viscachataenia quadrata (von Linstow, 1904) n. comb. (Cestoda: Anoplocephalidae) in Lagidium viscacia (Rodentia: Chinchillidae) from Argentina. Systematic Parasitology 54: 81-88.

Gómez L. A., M. T. López \& A. E. Gonzáles. 2008. Occurrence of Moniezia expansa (Rud., 1810) Blanchard, 1891 (Cestoda: Anoplocephalidae) in domestic pig (Sus scrofa domestica Linnaeus, 1758) in Peru. Veterinary Parasitology, 158: 380-381.

Parra, 1953. Estudio de dos nuevos helmintos intestinales de Lagidium peruanum. Publ. Mus. Hist. Nat. "Javier Prado" U.N.M.S.M. Ser. A Zoología No 11: 1-21.

Spasskii A. H. 1951. Anoplocephalate tapeworms of domestic and wild animals. Acad. Sci. U.R.S.S. Essentials of Cestodology. Vol. I. Moscow. Israel Prog. Scientific Transl. Jerusalem, 1961

Tenora F. 1976. Tapeworms of the family Anoplocephalidae Cholodkowsky, 1902. Evolutionary implications. Acta Sc. Nat. Brno 10: 1-37.

Zaldívar S. 1991. Zooparásitos de interés veterinario en el Perú. Ed. Maijosa. Lima, Perú. 252 pp. 